\title{
A simple device of generating glow discharge plasma in atmospheric pressure argon
}

\author{
Xuechen $\mathrm{Li}^{\mathrm{a}}{ }^{\mathrm{a}}$ Lifang Dong, Na Zhao, and Zengqian Yin \\ College of Physics Science and Technology, Hebei University, Baoding 071002, People's Republic of China \\ Tongzhen Fang and Long Wang \\ Institute of Physics, Chinese Academy of Sciences, Beijing 100080, People's Republic of China
}

(Received 6 September 2007; accepted 1 October 2007; published online 19 October 2007)

\begin{abstract}
Atmospheric pressure glow discharge is realized in argon by using a plasma needle. With increasing the applied voltage, uniform plasma increases in scale from a small region near the needle tip to a plasma plume with a length of about $20 \mathrm{~mm}$. The discharge mechanism is discussed based on the light emission waveforms from the plasma. Optical emission spectroscopy is used to determine excited electron temperature and vibrational temperature, and the results indicate that the excited electron temperature and the molecular vibrational temperature are about 6000 and $2300 \mathrm{~K}$, respectively. () 2007 American Institute of Physics. [DOI: 10.1063/1.2800814]
\end{abstract}

Research on atmospheric pressure glow discharges (APGDs) is motivated by applications such as thin film deposition, surface modification, sterilization, light sources, plasma display panels, plasma cloaking for aircraft, etc.

High-pressure glow discharges are prone to instabilities, which cause a transition from spatially uniform glow to an arc (filamentation). It must be damped. Much of the effort in generating stable APGD has focused on preventing the onset of instabilities. Multiple avalanches coupling at low breakdown voltage are necessary for generating APGD under sinusoidal voltage excitation. APGD has been investigated in helium and nitrogen theoretically and experimentally by numerous authors, ${ }^{1,2}$ and results show that interpulse preionization resulting from metastable particles can provide an additional source of primary electrons, which lowers breakdown voltage for a forthcoming half-cycle. Based on this viewpoint, ultraviolet radiation produced by spark discharges is employed by Qi et al. to supply preionization for realizing APGD. ${ }^{3}$ APGD is also realized in a device with a fine mesh inserted between the electrode and the barrier layer. Wang et al. explained that corona discharge around the mesh can provide interpulse electrons for APGD. ${ }^{4}$ An alternative approach for APGD is to restrict current growth by connecting a resistor or a semiconductor in series with the discharge device, though a pulse voltage on preventing the thermal instability is commonly employed to generate APGD at current densities above threshold for the glow-to-arc transition. ${ }^{7,8}$ An easy solution to avoid glow-to-arc transition at atmospheric pressure is to use microplasmas made in submillimetric scale. A plasma needle is proposed by Stoffels and co-workers ${ }^{9,10}$ which works under if conditions and plasma is stabilized by the flowing of working gas.

The focus of this paper is to present a plasma needle that is excited by a power source with a frequency of several tens of kilohertz. This simple and low cost device can generate APGD plasmas in stagnant argon and electric matching does not need to be considered. The basic aspects of this plasma needle are discussed and plasma parameters are given.

\footnotetext{
${ }^{\text {a) }}$ Author to whom correspondence should be addressed; electronic mail:
} xcli@hbu.cn
A schematic drawing of the plasma needle is shown in Fig. 1. Plasma is generated at the sharp end of a tungsten pin (tip radius can be adjusted from 70 to $100 \mu \mathrm{m}$, the experimental results are obtained with $90 \mu \mathrm{m}$ tip radius if no special explanation is given). The pin is fixed by a piece of rubber and it protruded from the holder by $3 \mathrm{~cm}$. The total length of the needle is about $5 \mathrm{~cm}$. The plasma needle is placed in a Perspex vacuum vessel. The vessel is pumped by a mechanical pump to its limit and then argon (99.99\%) is filled in to one atmospheric pressure. The blunt end of the needle is connected to the high voltage output of a power source. The frequency is fixed at $67 \mathrm{kHz}$. The applied voltage can be measured by a high voltage probe (Tektronix P6015A, 1000×) and recorded with an oscilloscope (Tektronix TDS3054B, $500 \mathrm{MHz}$ ). For recording the discharge images, a digital camera (Canon Powershot G1: 3.24 Mpixels) is used, and the exposure time is $8 \mathrm{~ms}$ in our experiments. Light emission is detected by a photomultiplier tube (RCA7265). Optical emission spectrum is obtained by a spectrometer equipped with a charge coupled device (CCD) detector (ACTON Research Spectropro2785, 1340 $\times 400$ pixels) with a grating of 2400 grooves $\mathrm{mm}^{-1}$. The entrance slit opening and the integration time of the CCD detector are maintained at $0.1 \mathrm{~mm}$ and $3 \mathrm{~ms}$, respectively.

Figure 2 shows a series of images of the discharge in atmospheric pressure argon with an increasing peak value of the applied voltage $\left(U_{p}\right)$. It can be found that the discharge is confined to the near region of the needle tip when $U_{p}$ is in the range from breakdown voltage to about $4200 \mathrm{~V}$. When

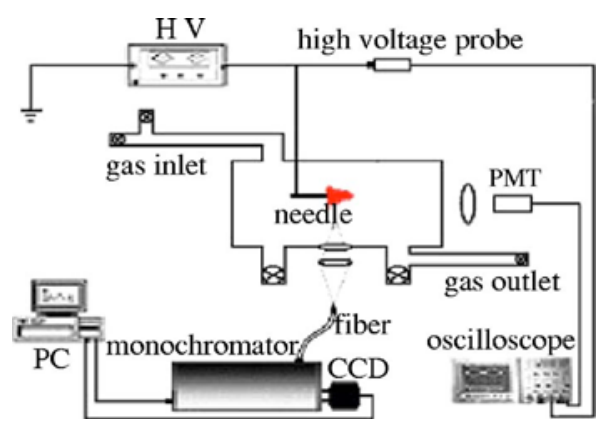

FIG. 1. (Color online) Schematic drawing of the experimental setup. 
(a)

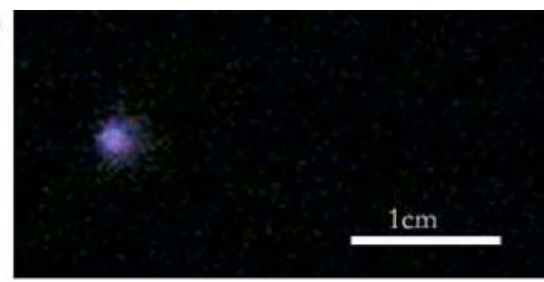

(b)

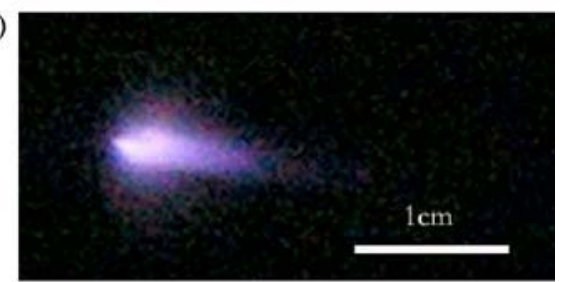

(c)

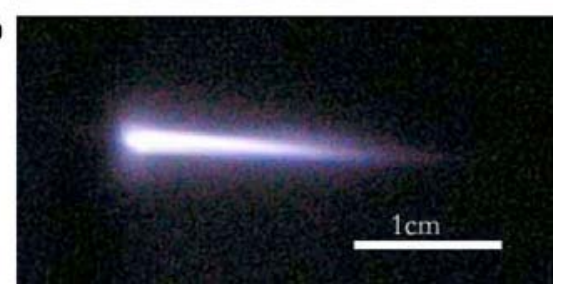

FIG. 2. (Color online) Discharge images under different $U_{p}$ : (a) $4 \mathrm{kV}$, (b) $6.5 \mathrm{kV}$, and (c) $9.5 \mathrm{kV}$.

$U_{p}$ exceeds $4200 \mathrm{~V}$, the scale of the plasma increases abruptly and shows a shape of a plume. With a further increase of $U_{p}$, the length of the plasma plume increases and the plasma turns brighter and brighter. Consequently, it can be found that the plasma needle has two discharge modes: corona discharge with $U_{p}$ below $4200 \mathrm{~V}$ and plasma plume discharge with $U_{p}$ above $4200 \mathrm{~V}$, respectively. Figure 3 indicates the scale of the plasma as a function of $U_{p}$. It is obvious that the scale of the plasma is kept constant (about $0.7 \mathrm{~mm}$ ) in corona discharge mode and increases with $U_{p}$ in plasma plume discharge mode. From Fig. 3, it can also be found that the plasma plume is longer when the tip radius is bigger, while the scale of the plasma is almost the same when the discharge is in corona discharge mode.

As shown in Fig. 4, the discharge in the corona mode consists of a series of pulses with width of about $200 \mathrm{~ns}$ [shown in Fig. 4(b)]. When $U_{p}$ is slightly above the breakdown voltage [shown in Fig. 4(a)], there is only one discharge pulse for 1 cycle of the applied voltage and the discharge pulse always appears at the negative half-cycle. As $U_{p}$ increases, the pulse number of negative half-cycle discharge also increases, as shown in Figs. 4(c)-4(e). The maximum number of discharge pulse is 5, and small light emission signal can be found at the positive half-cycle when $U_{p}$ is

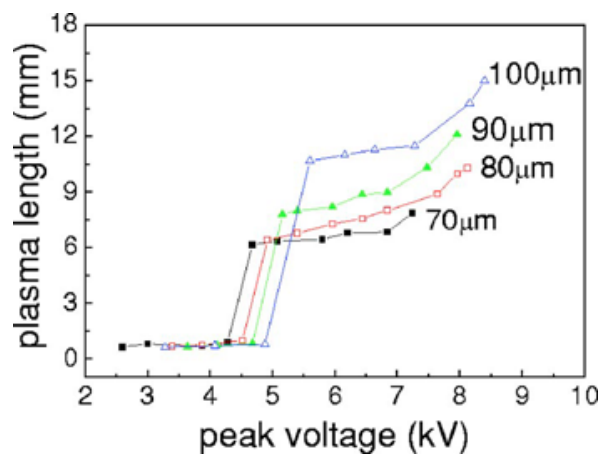

FIG. 3. (Color online) Plasma length as a function of $U_{p}$ and tip radius.
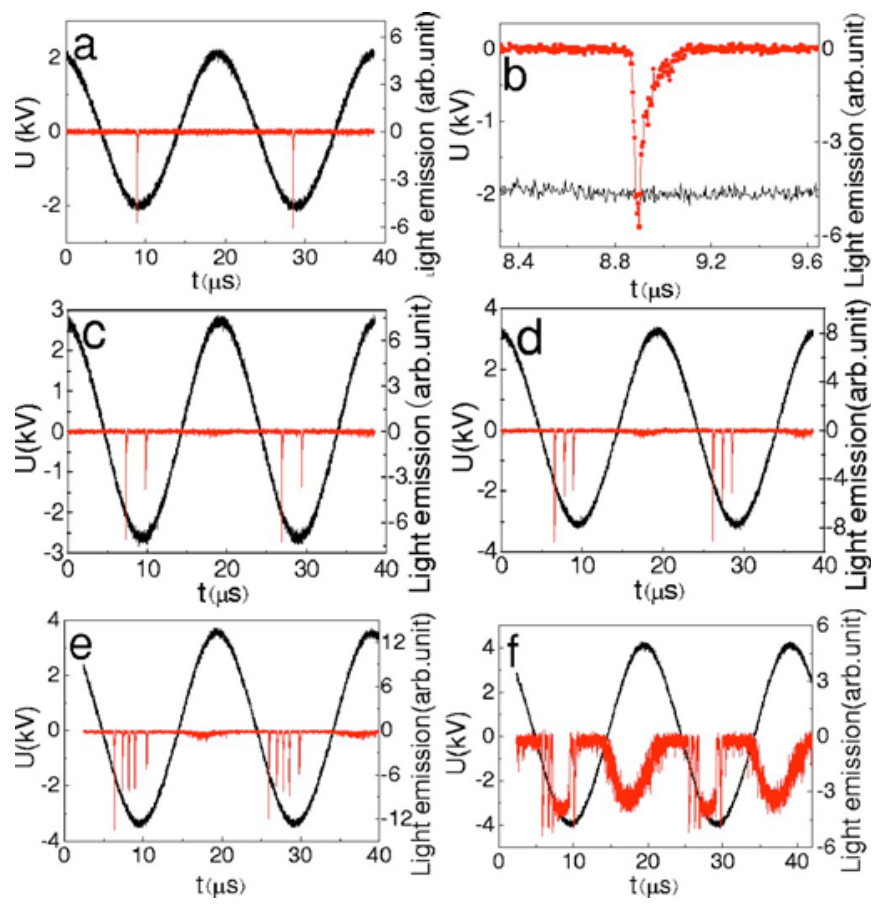

FIG. 4. (Color online) Waveforms of the applied voltage and light emission under different values of $U_{p}$. (b) is an enlarged part of (a). (a)-(e) correspond to the corona discharge mode shown in Fig. 2(a). (f) corresponds to that of a plasma plume.

high enough, as shown in Fig. 4(e). Figure 4(f) indicates the light emission for the plasma plume discharge mode. Obviously, a discharge hump (its width is about $7 \mu \mathrm{s}$ ) is shown at the positive half-cycle, while a hump (its width is about $2 \mu \mathrm{s})$ and some discharge pulses with width of about $200 \mathrm{~ns}$ are shown at the negative half-cycle.

The different waveforms may result from different discharge mechanisms for corona mode and plasma plume mode. In corona discharge mode, high-field region only exists at the vicinity near the needle tip. When the applied electric field is above critical value for breakdown, the number of electrons increases in the high-field region because of electron avalanche process. The drifting velocity of electrons is very low when the electrons move to the low-field region. Therefore, the negative potential needle tip is covered by an electron cloud. Because of shielding effect of this electron cloud, the electric field near the needle tip is lowered and the discharge is quenched. As a result, the light emission waveform shows a pulse. As time passes by, there are two factors for generating a consecutive discharge pulse. One is the increment of the applied voltage because a sinusoidal waveform is used. The other is that the electrons covering the needle tip move away because of slow drifting. Obviously, the discharge emission shows a series of short-lived pulses. Only discharge emission at the negative half-cycle is observed because the breakdown field for negative potential is lower than that for positive potential. However, when $U_{p}$ is high enough, as shown in Fig. 4(e), the electrons generated at the negative half-cycle will run toward the needle tip to excite neutral atoms, hence, little light emission signal appears at the positive half-cycle. The discharge mechanism at the corona discharge mode is similar to that of dc negative corona discharge. However, a different discharge mechanism is involved for the plasma plume discharge mode. In the plasma plume discharge, electrons move and accumulate at 

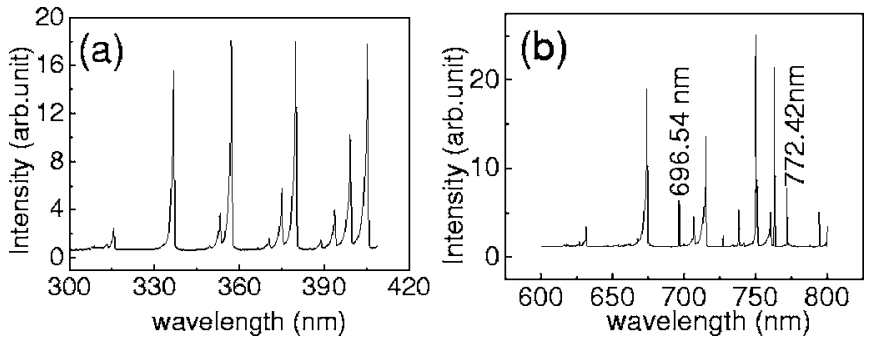

FIG. 5. Optical emission spectra in the range from (a) 300 to $410 \mathrm{~nm}$ and (b) 500 to $800 \mathrm{~nm}\left(U_{p}=6 \mathrm{kV}\right)$.

the tail of the plasma plume at the negative half-cycle. When the positive half-cycle comes, these accumulated electrons will move toward the needle tip and ionize neutral atoms to generate new electrons and positive ions. These positively charged ions will drift toward the plasma plume tail and accumulate there. Consequently, a virtual electrode is placed at the tail of the plasma plume. For the negative half-cycle discharge, electrons accumulate on this virtual electrode, while for positive half-cycle discharge, electrons emit from this electrode and positive ions accumulate on this electrode. Because of this virtual electrode (spatial charges), the electric potential distribution is different from that in the corona discharge and the electric field in the whole gap between the needle tip and the virtual electrode is high enough to form a glow discharge. The virtual electrode is not grounded, so glow-to-arc transition is damped. Therefore, a high-pressure glow discharge is generated in the gap between the needle tip and the virtual electrode.

Figures 5(a) and 5(b) indicate the optical emission spectra in the range from 300 to $410 \mathrm{~nm}$ and 500 to $800 \mathrm{~nm}$, respectively. The intensity of the second positive system of $\mathrm{N}_{2}$ is used to calculate the molecular vibrational temperature. ${ }^{1 \mathrm{~T}}$
The spectral lines, 687.13, 696.54, and $772.42 \mathrm{~nm}$, of argon are chosen to determine the excited electron temperature using a Boltzmann plot. ${ }^{12}$ The results show that the molecular vibrational temperature is about $2300 \mathrm{~K}$ and the excited electron temperature is about $6000 \mathrm{~K}$ for the plasma plume discharge mode. These plasma parameters are functions of the experimental parameters such as $U_{p}$, frequency, distance from the tip, etc. More detailed work is under research and will be published elsewhere.

This paper was sponsored by the National Natural Science Foundation of China under Grant Nos. 10575027 and 10647123, and the National Natural Science Foundation of Hebei province, China under Grant Nos. A2007000134 and A2006000950.

${ }^{1}$ S. Kanazawa, M. Kogoma, T. Moriwaki, and S. Okazaki, J. Phys. D 21, 838 (1988)

${ }^{2}$ F. Massines, N. Gherardi, N. Naude, and P. Segur, Plasma Phys. Controlled Fusion 47, B577 (2005).

${ }^{3}$ B. Qi, C. S. Ren, D. Z. Wang, S. Z. Li, K. Wang, and Y. T. Zhang, Appl. Phys. Lett. 89, 131503 (2006).

${ }^{4}$ X. X. Wang, H. Y. Luo, Z. Liang, T. Mao, and R. L. Ma, Plasma Sources Sci. Technol. 15, 845 (2006).

${ }^{5}$ A. Risacher, S. Larigaldie, G. Bobillot, J. P. Marcellin, and L. Picard, Plasma Sources Sci. Technol. 16, 200 (2007).

${ }^{6}$ Y. Yang, IEEE Trans. Plasma Sci. 31, 174 (2003).

${ }^{7}$ J. L. Walsh, J. J. Shi, and M. G. Kong, Appl. Phys. Lett. 89, 161505 (2006).

${ }^{8}$ K. Takaki, M. Hosokawa, T. Sasaki, S. Mukaigawa, and T. Fujiwara, Appl. Phys. Lett. 86, 151501 (2005).

${ }^{9}$ E. P. van der Laan, E. Stoffels, and M. Steinbuch, Plasma Sources Sci. Technol. 15, 582 (2006).

${ }^{10}$ I. E. Kieft, E. P. van der Laan, and E. Stoffels, New J. Phys. 6, 149 (2004).

${ }^{11}$ G. Herzberg, Molecular Spectra and Molecular Structure (Science, Beijing, 1983), Vol. I, pp. 273-275.

${ }^{12}$ S. Forster, C. Mohr, and W. Viol, Surf. Coat. Technol. 200, 827 (2005). 